\title{
Effect of iodine-therapy on hyperthyroidism patients without pre-administration of anti-thyroid therapeutic options
}

${ }^{1}$ Associate Professor, Diabetes Research Center, Health Research Institute, Ahvaz Jundishapur University of Medical Sciences, Ahvaz, Iran.

${ }^{2}$ Assistant Professor, Diabetes Research Center, Health Research Institute, Ahvaz Jundishapur University of Medical Sciences, Ahvaz, Iran.

${ }^{3}$ Department of Statistics and Epidemiology, Faculty of Public Health, Diabetes Research Center, Ahvaz Jundishapur University of Medical Sciences, Ahvaz, Iran. Corresponding author: naimiz@ymail.com.

Abstract: Anti-thyroid treatment and radioiodine are mainly used treatments for patients with thyrotoxicosis. The effect of anti-thyroid drugs (ATDs) on radioactive iodine's therapeutic effectiveness in hyperthyroidism remains controversial. This study aimed to determine the treatment effectiveness and complications in patients with thyrotoxicosis receiving radioiodine treatment with or without adjunctive anti-thyroid drugs in a tertiary endocrinology center in Ahvaz, Iran. A total of 165 patients with thyrotoxicosis (50 males and 115 females) were recruited in this retrospective clinical study. These patients were divided into two groups: one group underwent natural iodine therapy without being treated with anti-thyroid drugs ( $\mathrm{N}=86)$, and the other group was first treated with anti-thyroid drugs and then treated with iodine $(\mathrm{N}=79)$. Then, the frequency of euthyroidism, thyroid storm, thyroiditis, hypothyroidism and the time to reach euthyroidism is evaluated. The results showed no difference between the two groups when reaching the euthyroid and the mean numerical mean TSH at the time of hypothyroidism. However, the average dose of iodine received in the concomitant drug group was lower. No thyroid storm was observed in the two groups regarding complications after treatment. Also, the prevalence of hypothyroidism and thyroiditis were not significantly different between the two groups. lodine therapy with and without the use of anti-thyroid drugs (ATD) in low-risk patients with thyrotoxicosis has no difference in treatment outcomes, response to treatment, and subsequent complications.

Key words: Anti-thyroid drugs, radioactive iodine (I131), lodine therapy, thyrotoxicosis.

\section{Introduction}

Thyrotoxicosis is an excess thyroid hormone condition, a common disease, especially in women. The most common cause of thyrotoxicosis is Graves' disease. Other causes include toxic nodular hyperthyroidism and thyroiditis ${ }^{1,2}$. Common signs of disease are fatigue, weight loss, tremor, anxiety, palpitations, disturbed sleep, sweating, heat intolerance, and polydipsia ${ }^{3,4}$. The therapeutic possibilities for thyrotoxicosis treatment for these conditions, anti-thyroid drugs (ATD), thyroidectomy, and radioactive iodine $\mathrm{I}^{131}$ (RAI)..$^{5-7}$ early and timely treatment of hyperthyroidism also may prevent the progress of the disease ${ }^{8}$.

Each of the treatment modalities has particular efficacy limitations and potential adverse effects ${ }^{9,10}$. The main clinical downside of ATD therapy is the high rate of hyperthyroidism recurrence when therapy is stopped ${ }^{11-16}$. However, the ease of ATD treatment and the fact that the thyroid is not irreversibly compromised has rendered long-term, low-dose ATD treatment a rational choice for disease control ${ }^{11}$. Radioactive iodine therapy is an essential definitive treatment of hyperthyroidism, used for more than six decades as first-line or second-line therapy, and $1^{131}$ therapy is cost-effective, safe, and reliable ${ }^{5,12}$. This data would be a valuable resource for physicians to involve patients in the shared decision-making process ${ }^{13}$. The effect of anti-thyroid drugs (ATDs) on radioactive iodine's therapeutic effectiveness in hyperthyroidism remains controversial ${ }^{14}$. So far, no study has been conducted in our country and this epi- demiological region regarding the outcomes, results, and incidence of each of the possible complications in iodine therapy alone with and without anti-thyroid drug treatment of patients with thyrotoxicosis. This study aimed to assess the treatment effectiveness in patients with thyrotoxicosis receiving radioiodine treatment with or without adjunctive anti-thyroid drugs in a tertiary endocrinology center in Ahvaz, Iran.

\section{Materials and methods}

\section{Ethics}

The personal information of included individuals was kept secret. The ethical council approved the survey.

\section{Inclusion criteria}

According to our inclusion criteria, a total of 165 patients with thyrotoxicosis (50 males and 115 females) were recruited in the retrospective clinical study. Data on consecutive patients between 2015 to 2020 at the Endocrinology Clinics (Ahvaz, Iran) were retrospectively collected and reviewed. The diagnosis of thyrotoxicosis was performed according to the basis of elevated total T4 (TT4) and/or total T3 (TT3) values and TSH suppressed to $<0.01 \mathrm{mlU} / \mathrm{L}^{15}$.

Citation: Rashidi H, Ghaderian B, Sedaghat A, Latifi M, Naimi Z. Effect of iodine-therapy on hyperthyroidism patients without pre-administration of anti-thyroid therapeutic options. Revis Bionatura 2022;7(1). 7. http://dx.doi.org/10.21931/RB/2022.07.01.7

Received: 25 June 2021 / Accepted: 10 August 2021 / Published: 15 February 2022

Publisher's Note: Bionatura stays neutral with regard to jurisdictional claims in published maps and institutional affiliations. 


\section{Groups}

All cases were divided into two groups: one group receiving the drug before iodine was considered $(n=86)$, and the other group receiving only the iodine was defined $(n=79)$. Inclusion Criteria were age between 18 to 65 years old and absence of comorbid diseases. Individuals over 65 years, patients with heart failure (Ejection fraction $<40 \%$ ), pregnant women were excluded from this study. At the diagnosis and approximately 2 months intervals, serum- triiodothyronine (T3), thyroxine (T4), and TSH levels were analyzed.

These patients were divided into two groups: one group underwent natural iodine therapy without being treated with anti-thyroid drugs ( $\mathrm{N}=86$ ). The other group was first treated with anti-thyroid drugs and then with iodine $(\mathrm{N}=79)$. Then, the frequency of thyroid storm, thyroiditis and hypothyroidism, euthyroidism and the time to reach euthyroidism was examined using their evaluation. Euthyroidism was defined as T3, T4 serum levels within the normal range.

\section{Questionnaire}

A questionnaire was designed to retrospectively obtain all demographic, clinical, laboratory, and radiological results from the patients' medical records. All the experiments were performed according to the Ethical Committee's guidelines at the Ahvaz Jundishapur University of Medical Sciences, and all participants provided written informed consent.

\section{Statistical analysis}

Statistical analysis was performed using SPSS software (SPSS Inc, version 23, Chicago, IL, USA). Kolmogorov-Smirnov, and Shapiro-Wilk tests were used to test for the data normality. Central and descriptive statistics were reported for quantitative data. A Chi-square test was used to compare the ratio of complications in qualitative variables. P-value $<0.05$ was considered statistically significant ${ }^{16-19}$.

\section{Results}

\section{Demographic characteristics}

The median age in the iodine alone group and iodine with the drug group was $47.22 \pm 15.7$ and $44.07 \pm 12.38$. There was no statistically significant difference between the median ages of the two groups $(\mathrm{P}=0.15)$. The detail of the demographic characteristics of the study population is indicated in Table 1.

\section{Comparison of the frequency of complications}

Regarding the occurrence of complications after treatment, no thyroid storm was observed between the two groups, and the prevalence of hypothyroidism and thyroiditis were not significantly different between the two groups (Table 2).

The results showed no difference between the two groups when reaching the euthyroidism and the mean TSH at the time of hypothyroidism ( $P=0.11$ and $P=0.14$, respectively).

\section{Comparison time to euthyroidism \& iodine dose}

However, there was a significant difference in lodine dose between the two groups ( $P=0.005)$, and the average dose of iodine received in the iodine with drug therapy group was lower than in the iodine alone group. The level of T3 at the beginning of the study was not significantly different between the two groups. However, the level of T4, which was significantly higher in the iodine alone group at the beginning of the study, showed a more significant decrease at the end of the treatment period than the iodine with drug therapy groups (Table 3 ).

The regression equation was used to find the correlation between levothyroxine administration and several variables.

\section{Results of the regression equation}

There was no significant correlation between that time and first T3, T4 variables, lodine dose, thyrotoxicosis syndro-

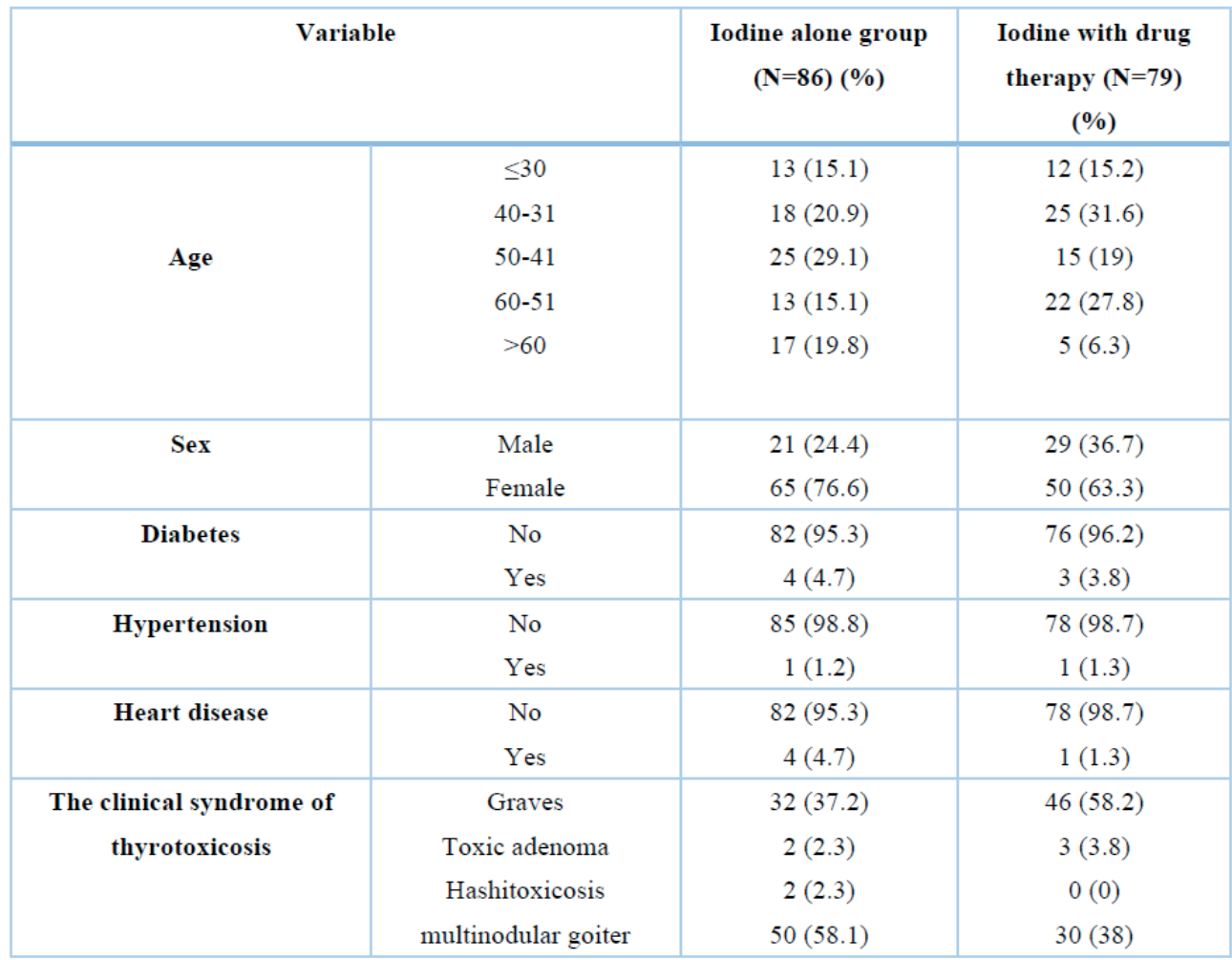

Table 1. Demographic Characteristics of the study population. 


\begin{tabular}{|c|c|c|c|c|}
\hline \multicolumn{2}{|c|}{ Variable } & Iodine alone group (\%) & Iodine with drug therapy (\%) & P-value \\
\hline Hypothyroidism & No & $31(36.0)$ & $31(39.2)$ & 0.67 \\
& Yes & $55(64.0)$ & $48(60.8)$ & 0.47 \\
\hline Thyroiditis & No & $86(100.0)$ & $78(98.7)$ & $1(1.3)$ \\
& Yes & $0(0.0)$ & \\
\hline
\end{tabular}

Table 2. Comparison of the frequency of complications in the two groups.

\begin{tabular}{|c|c|c|c|}
\hline Variable & Iodine therapy group (mean \pm SD) & Iodine with drug therapy group (mean \pm SD) & P-value \\
\hline Euthyrodism Day & $68.72 \pm 60.58$ & $60.58 \pm 30.31$ & 0.11 \\
\hline Iodine dose & $23.8 \pm 8.7$ & $19.86 \pm 9.21$ & $0.005^{*}$ \\
\hline
\end{tabular}

Table 3. Comparison time to euthyroidism \& iodine dose between the two groups.

\begin{tabular}{|c|c|c|c|}
\hline Variable & B & SD (Error) & P-value \\
\hline T3 (first) & 0.17 & 2.34 & 0.94 \\
\hline T4 (first) & -1.05 & 1.17 & 0.374 \\
\hline Iodine cumulative dose & 0.004 & 1.21 & 0.99 \\
\hline Times of iodine therapy & -67.22 & 76.02 & 0.38 \\
\hline Euthyrodism day & 0.76 & 0.32 & 0.024 \\
\hline Type of thyrotoxicosis Syndrome & 12.23 & 8.64 & 0.16 \\
\hline Age & -1.33 & 0.69 & 0.062 \\
\hline Sex & -22.46 & 22.73 & 0.32 \\
\hline
\end{tabular}

Table 4. The results of the regression equation.

me, age, and sex $(P>0.05)$. At the same time, Euthyroidism time showed a statistically significant correlation with the Levoday variable (levothyroxine onset time) $(P=0.024)$ (Table 4).

\section{Discussion}

Several developments have been occurred in medical sciences ${ }^{20-25}$. However, there were so many defects regarding knowledge about diseases and their treatments ${ }^{26-34}$. lodine therapy is the definitive treatment modality of hyperthyroidism ${ }^{35}$; however, several concerns exist regarding this approach's efficacy $^{36}$. McDermott et al. believed that the possibility of thyroid dysfunction increases upon iodine therapy ${ }^{37}$. This mainly occurs in high-risk patients such as the elderly, individuals with underlying heart diseases, and patients with enlarged thyroids. Therefore, these patients should undergo an anti-thyroid regimen before iodine therapy. However, our results have shown that iodine therapy alone could be safe with minimum side effects while generating similar treatment outcomes ${ }^{37}$. In our study, no difference was observed in adverse effects such as thyroid storm, hypothyroidism and thyroiditis between the two study groups. The time point of achieving euthyroidism served as an independent and strong predictive factor for hypothyroidism development. This means that other variables, including age, gender, type of clinical syndrome, the initial number of T3 and T4, were not considered for predicting the development of hypothyroidism and initiation of Levothyroxine. Some studies have shown that ATDs can have a protective effect, which leads to a decrease in the thyroid gland's effective half-life and radioactive iodine uptake ${ }^{38,39}$. A meta-analysis study investigated the effect of utilizing anti-thyroid drugs before iodine therapy and compared it with iodine therapy alone in grave patients.
Results have shown that administrating anti-thyroid drugs before iodine therapy increases treatment failure yet decreases subsequent hypothyroidism ${ }^{40}$. Our results were not similar to those of the previous survey since we did not observe a difference in treatment responses, euthyroidism, and even subsequent hypothyroidism between them. The discrepancy could be explained as follow: First, in the mentioned study, the administered drug was PTU, while in our study, different types of drugs, and mostly methimazole, were utilized. In addition, Grave's disease was the only thyrotoxicosis syndrome evaluated in the mentioned study, but in our study, several thyrotoxicosis syndromes were assessed. Therefore, a separate study on Grave's patients treated with methimazole is suggested to evaluate the inconsistency of these results and investigate the effect of a special type of drug on the particular type of clinical syndrome.

Ding et $a l^{41}$ conducted a study to evaluate long-term outcomes and immunity of iodine therapy without drug therapy. In their study, of 408 patients, 283 individuals were treated with iodine therapy, 15 of whom achieved complete remission and 47 partial remissions. Besides, no signs of disease relapse and recurrence were observed in patients treated with iodine alone. This study concluded that utilizing iodine therapy alone is safe and effective in treating patients with severe hyperthyroidism who are resistant to anti-thyroid drugs ${ }^{41}$. Similarly, our results have shown that administrating iodine therapy alone is safe in patients with thyrotoxicosis and, in terms of adverse effects, is similar to the combination therapy of drug and iodine therapy. In Ding et al.'s study, the follow-up period was longer, and the sample size was larger. Moreover, patients with heart disease and arrhythmia, hepatic dysfunctions, and over 65. Nevertheless, we excluded patients over 65 years and those with underlying heart disorders since they were considered a 
high-risk group to treat using the iodine therapy without concomitant ATDs therapy.

There are several contradictions regarding the effect of anti-thyroid drugs and their impact on iodine therapy. Studies have shown that utilizing PTU (and not methimazole) before iodine therapy delays the therapeutic response. PTU is suggested to enhance thyroid tissue resistance against radioactive iodine ${ }^{5,42-44}$. In a meta-analysis study performed on 1269 patients, drug therapy's risk of reducing the response to iodine therapy was approximately 1.28. After iodine therapy, the effect of hypothyroidism was not related to the type of drug used $^{40}$. The results of this meta-analysis were different from our study. Anti-thyroid drug treatment before iodine therapy did not affect the treatment results and subsequent complications in the current study.

Our study's limitation was the inclusion of low-risk patients in the study. In other words, it is unclear whether iodine therapy alone before ATD will have the same results in high-risk patients, such as the elderly, diabetic patients, and underlying heart disease. One of the study's strengths was evaluating different types of thyrotoxicosis syndromes, while most studies have been done on Graves' disease. The current study investigated the relations between $T 4$, frequency of iodine therapy, iodine dose, time of euthyroidism, and hypothyroidism, which was for the first time. Our findings recommend comparing iodine therapy alone or ATD treatment before iodine therapy, with different drugs separately and in different thyrotoxicosis syndromes separately with a more significant number of subjects.

\section{Conclusions}

lodine therapy with and without the anti-thyroid drug treatment in low-risk patients with thyrotoxicosis has no difference in therapy outcomes, response to treatment, and subsequent complications. The hypothyroidism time depends on the time of euthyroidism after receiving iodine in two groups. More quality randomized controlled trials are necessary to evaluate the quality of life, cost of the two treatments and more attention to confounding factors such as goiter size and thyroid uptake is recommended.

\section{Acknowledgments}

The authors would like to thank the staff of the Diabetes Research Center, Health Research Institute, Ahvaz Jundishapur University of Medical Sciences, Ahvaz, Iran.

\section{Bibliographic references}

1. Franklyn JA, Boelaert K. Thyrotoxicosis. The Lancet. 2012;379(9821):1155-66.

2. Burch HB, Cooper DS. Management of Graves disease: a review. Jama. 2015;314(23):2544-54.

3. Goichot B, Caron P, Landron F, Bouée S. Clinical presentation of hyperthyroidism in a large representative sample of outpatients in France: relationships with age, aetiology and hormonal parameters. Clinical endocrinology. 2016;84(3):445-51.

4. Pearce EN. Diagnosis and management of thyrotoxicosis. Bmj. 2006;332(7554):1369.

5. lagaru A, McDougall IR. Treatment of thyrotoxicosis. Journal of nuclear medicine. 2007;48(3):379-89.

6. Wartofsky L, Glinoer D, Solomon B, Nagataki S, Lagasse R, Nagayama $Y$, et al. Differences and similarities in the diagnosis and treatment of Graves' disease in Europe, Japan, and the United States. Thyroid. 1991;1(2):129-35.
7. Brito JP, Schilz S, Singh Ospina N, Rodriguez-Gutierrez R, Maraka $\mathrm{S}$, Sangaralingham LR, et al. Anti-thyroid drugs-the most common treatment for Graves' disease in the United States: a nationwide population-based study. Thyroid. 2016;26(8):1144-5.

8. Ross DS. Radioiodine therapy for hyperthyroidism. New England Journal of Medicine. 2011;364(6):542-50.

9. Smith TJ, Hegedüs L. Graves' disease. New England Journal of Medicine. 2016;375(16):1552-65.

10.Eliasson $M$. Long-term prognosis after medical treatment of Graves' disease in a northern Swedish population 2000-2010. Endocrinology. 2014;170:419-27.

11. Orgiazzi J. Should protracted treatment with anti-thyroid drug (ATD) be considered as a routine strategy in patients with graves' disease who had a relapse after a first course of ATD? Clinical Thyroidology. 2015;27(11):302-5.

12. Silberstein EB, Alavi A, Balon HR, Clarke SE, Divgi C, Gelfand MJ, et al. The SNMMI practice guideline for therapy of thyroid disease with 131I 3.0. Journal of Nuclear Medicine. 2012;53(10):1633-51.

13. Azizi F, Malboosbaf R. Long-term anti-thyroid drug treatment: a systematic review and meta-analysis. Thyroid. 2017;27(10):1223-31.

14. Kartamihardja AHS, Massora S. The Influence of Antithyroid Drug Discontinuation to the Therapeutic Efficacy of 131 in hyperthyroidism. World journal of nuclear medicine. 2016;15(2):81.

15. Koulouri O, Moran C, Halsall D, Chatterjee K, Gurnell M. Pitfalls in the measurement and interpretation of thyroid function tests. Best practice \& research Clinical endocrinology \& metabolism. 2013;27(6):745-62.

16. Ghorbani F, Gheisari E, Dehkordi FS. Genotyping of vacA alleles of Helicobacter pylori strains recovered from some Iranian food items. Trop J Pharm Res. 2016;15(8):1631-6.

17. Nejat S, Momtaz H, Yadegari M, Nejat S, Safarpour Dehkordi F, Khamesipour F. Seasonal, geographical, age and breed distributions of equine viral arteritis in Iran. Kafkas Univ Vet Fak Derg. 2015;21(1):111-6.

18. Dehkordi FS, Momtaz H, Doosti A. Application of Real-Time PCR for detection of Aspergillus species in aborted ruminant foetuses. BulgaJ Vet Med. 2012;15(1):30-6.

19. Dehkordi, F.S., Saberian, S., Momtaz, H. Detection and segregation of Brucella abortus and Brucella melitensis in Aborted Bovine, Ovine, Caprine, Buffaloes and Camelid Fetuses by application of conventional and real-time polymerase chain reaction. The Thai J Vet Med. 2012;42(1):13.

20.Dehkordi FS. Prevalence study of Coxiella burnetii in aborted ovine and caprine fetuses by evaluation of nested and real-time PCR assays. American J Anim Vet Sci. 2011;6(4):180-6.

21. Dehkordi FS, Tirgir F, Valizadeh Y. Effects of Guajol ${ }^{\circledR}$ ointment synthesized from medicinal smoke condensate of jennet feces on burn wound healing on Wistar rat. Vet Res Forum. 2017; 8(3):215.

22. Dehkordi FS, Tavakoli-Far B, Jafariaskari S, Momtaz H, Esmaeilzadeh S, Ranjbar R, Rabiei M. Uropathogenic Escherichia coli in the high vaginal swab samples of fertile and infertile women: virulence factors, O-serogroups, and phenotyping and genotyping characterization of antibiotic resistance. New Microb New Infect. 2020;38:100824.

23. Safarpordehkordi F, Yahaghi E, Khodaverdi Darian E. Prevalence of antibiotic resistance in Escherichia coli isolated from poultry meat supply in Isfahan. Iran J Med Microbiol. 2014;8(2):41-7.

24. Dehkordi FS, Haghighi N, Momtaz H, Rafsanjani MS, Momeni M. Conventional vs real-time PCR for detection of bovine herpes virus type 1 in aborted bovine, buffalo and camel foetuses. Bulgar J Vet Med. 2013;16(2):102-12.

25. Dehkordi FS, Yazdani F, Mozafari J, Valizadeh Y. Virulence factors, serogroups and antimicrobial resistance properties of Escherichia coli strains in fermented dairy products. BMC Res Notes. 2014;7(1):1-8.

26. Dehkordi FS, Barati S, Momtaz H, Ahari SN, Dehkordi SN. Comparison of shedding, and antibiotic resistance properties of Listeria monocytogenes isolated from milk, feces, urine, and vaginal secretion of bovine, ovine, caprine, buffalo, and camel species in Iran. Jundishapur J Microbiol. 2013;6(3):284. 
27. Safarpour Dehkourdi F, Momtaz H, Esmailzade S, Khayyat Khameneie M, Yahaghi E. Detection of virulence factors of Uropathoigenic Escherichia coli isolates from infertile women high vaginal swabs. Iran J Med Microbiol. 2014;7(4):1-8.

28. Dehkordi FS, Gandomi H, Basti AA, Misaghi A, Rahimi E. Phenotypic and genotypic characterization of antibiotic resistance of methicillin-resistant Staphylococcus aureus isolated from hospital food. Antimicrob Res Infect Control. 2017;6(1):1-1.

29. Dehkordi FS. Prevalence study of Bovine viral diarrhea virus by evaluation of antigen capture ELISA and RT-PCR assay in Bovine, Ovine, Caprine, Buffalo and Camel aborted fetuses in Iran. AMB Express. 2011;1(1):1-6.

30.Dehkordi FS, Parsaei P, Saberian S, Moshkelani S, Hajshafiei P, Hoseini SR, Babaei M, Ghorbani MN. Prevalence study of Theileria annulata by comparison of four diagnostic Techniques in shouthwest Iran. Bulgar J Vet Med. 2012;15(2): 123-130.

31. Dehkordi FS, Haghighi Borujeni MR, Rahimi E, Abdizadeh R. Detection of Toxoplasma gondii in raw caprine, ovine, buffalo, bovine, and camel milk using cell cultivation, cat bioassay, capture ELISA, and PCR methods in Iran. Foodborne Pathog Dis. 2013;10(2):120-5.

32. Dehkordi FS, Khamesipour F, Momeni M. Brucella abortus and Brucella melitensis in Iranian bovine and buffalo semen samples: The first clinical trial on seasonal, Senile and geographical distribution using culture, conventional and real-time polymerase chain reaction assays. Kafkas Univ Vet Fak Derg. 2014;20(6):821-8.

33. Dehkordi FS, Valizadeh Y, Birgani TA, Dehkordi KG. Prevalence study of Brucella melitensis and Brucella abortus in cow's milk using dot enzyme linked immuno sorbent assay and duplex polymerase chain reaction. J Pure Appl Microbiol. 2014;8(2):1065-9.

34. Safarpour Dehkordi F, Hosseini S, Rahimi E, Momeni M, Yahaghi E, Khodaverdi Darian E. Investigate the frequency of virulence genes Vibrio parahaemolyticus isolated from fish, lobsters and crabs caught from Persian Gulf. Iran J Med Microbiol. 2014;8(2):1-7.

35. Ma C. The long term effects of radioiodine therapy versus anti-thyroid medications on Graves' disease. Journal of Nuclear Medicine. 2013;54(supplement 2):40-.
36. Braga M, Walpert N, Burch HB, Solomon BL, Cooper DS. The effect of methimazole on cure rates after radioiodine treatment for Graves' hyperthyroidism: a randomized clinical trial. Thyroid. 2002;12(2):135-9.

37. McDermott MT, Kidd GS, Dodson Jr LE, Hofeldt FD. Radioiodine-induced thyroid storm: case report and literature review. The American journal of medicine. 1983;75(2):353-9.

38. Moka D, Dietlein M, Schicha H. Radioiodine therapy and thyrostatic drugs and iodine. European journal of nuclear medicine and molecular imaging. 2002;29(2):S486-S91.

39. Mumtaz M, Lin LS, Hui KC, Khir ASM. Radioiodine I-131 for the therapy of Graves' disease. The Malaysian journal of medical sciences: MJMS. 2009;16(1):25.

40.Walter MA, Briel M, Christ-Crain M, Bonnema SJ, Connell J, Cooper DS, et al. Effects of anti-thyroid drugs on radioiodine treatment: systematic review and meta-analysis of randomised controlled trials. Bmj. 2007;334(7592):514.

41. Ding $Y$, Xing J, Fang $Y$, Wang $Y$, Zhang $Y$, Long $Y$. 131I therapy for 345 patients with refractory severe hyperthyroidism: Without anti-thyroid drug pretreatment. Experimental Biology and Medicine. 2016;241(3):290-5.

42. Medghalchi, A., Akbari, M., Alizadeh, Y., Moghadam, R.S. The epidemiological characteristics of patients with thyroid eye disease in a referral center in northern Iran. Journal of Current Ophthalmology, 30(4), pp. 353-358

43.Imseis RE, Vanmiddlesworth L, Massie JD, Bush AJ, Vanmiddlesworth N. Pretreatment with propylthiouracil but not methimazole reduces the therapeutic efficacy of iodine- 131 in hyperthyroidism. The Journal of Clinical Endocrinology \& Metabolism. 1998;83(2):685-7.

44.Weaver JU, Razvi S. Dose of anti-thyroid drugs can effect remission in Grave's disease. Endocrine journal. 2005;52(1):159- 